\title{
A Taxonomic Study of Sclerotinia sclerotiorum and Related Species: Mycelial Interactions
}

\author{
By A.-L. WONG AND H. J. WILLETTS \\ School of Botany, University of New South Wales, Kensington, \\ N.S.W. 2033, Australia
}

(Received 26 November 1974; revised 23 January 1975)

\begin{abstract}
SUMMARY
Selected isolates of Sclerotinia sclerotiorum (Lib.) de Bary were grown together on agar, and the mycelial reactions in the regions of association studied. Three types of reaction were observed: (i) free intermingling of mycelia without antagonism and with occasional hyphal anastomoses; (ii) a white, later pigmented, incompatibility zone associated with excessive branching of hyphal tips at the margins of one or both of the colonies; (iii) a brown incompatibility zone of variable intensity, accompanied by early lysis of the tips of the peripheral hyphae of one of the colonies. Associated with reaction types (ii) and (iii) were interactions in which the hyphae of one isolate attached themselves to and grew around those of another isolate; this resulted in the lysis of the 'attacked' hyphae. The grouping of the selected isolates by means of reaction types and 'predatory' interactions gives support for the separation of $S$. sclerotiorum into three distinct species, $S$. minor, S. trifoliorum and S. sclerotiorum.
\end{abstract}

\section{INTRODUCTION}

In the past there has been much controversy regarding the taxonomy of Sclerotinia sclerotiorum (Lib.) de Bary [= Whetzelinia sclerotiorum (Lib.); Korf \& Dumont, 1972], mainly because of the inadequacy of morphological criteria. Some workers (Jagger, I920; Keay, 1939; Williams \& Western, 1965) recognized $S$. sclerotiorum, $S$. trifoliorum and $S$. minor as separate species, but Purdy (I955) concluded that there was insufficient evidence to justify this separation and included them all in the one species, $S$. sclerotiorum. In recent work we have used ontogenetic (Willetts \& Wong, I97I) and electrophoretic (Wong \& Willetts, 1973) techniques to study the taxonomy of this group of fungi. Our findings have provided further evidence for the existence of three distinct species.

Reinhardt (I892) found, from co-inoculation experiments, that $S$. trifoliorum was incompatible with $S$. tuberosa and that the hyphae of the former appeared to attack those of $S$. tuberosa. He concluded that this antagonism between the mycelia indicates specific differences. Björling (I95I) found that mycelia of different isolates of $S$. trifoliorum were compatible, but Loveless (I95I) observed a pigmented 'incompatibility line' between $S$. trifoliorum isolates from pasture legumes and others from Vicia faba. He used this as a criterion to separate $S$. trifoliorum var. fabae from $S$. trifoliorum. We sought further information on the taxonomy of this group of fungi by studying the mycelial interactions of selected isolates. 
Table I. Details of isolates of Sclerotinia spp.

\begin{tabular}{|c|c|c|c|}
\hline $\begin{array}{l}\text { Isolate } \\
\text { reference } \\
\text { code }\end{array}$ & $\begin{array}{l}\text { Host from } \\
\text { which } \\
\text { isolated }\end{array}$ & $\begin{array}{c}\text { Size of } \\
\text { sclerotium } \\
\text { in culture }\end{array}$ & $\begin{array}{c}\text { Name given } \\
\text { at time of } \\
\text { isolation }\end{array}$ \\
\hline $\begin{array}{l}\text { SM-P } \\
\text { SM-T } \\
\text { SM-SI } \\
\text { SM-S2 }\end{array}$ & $\left.\begin{array}{l}\text { Potato } \\
\text { Tomato } \\
\text { Sunflower } \\
\text { Sunflower }\end{array}\right\}$ & $\begin{array}{l}\text { Small } \\
\left(0 \cdot 3^{-2} \cdot 0 \mathrm{~mm} \text { diam. }\right)\end{array}$ & Sclerotinia minor \\
\hline $\begin{array}{l}\text { ST-LI } \\
\text { ST-L2 } \\
\text { ST-B I } \\
\text { ST-B2 }\end{array}$ & $\left.\begin{array}{l}\text { Lucerne } \\
\text { Lucerne } \\
\text { French bean } \\
\text { French bean }\end{array}\right\}$ & $\begin{array}{l}\text { Large } \\
(\mathrm{I} \cdot 0-8 \cdot 0 \mathrm{~mm} \text { diam. })\end{array}$ & S. trifoliorum \\
\hline $\begin{array}{l}\text { SS-CLI } \\
\text { SS-CB } \\
\text { SS-CP } \\
\text { SS-SU }\end{array}$ & $\left.\begin{array}{l}\text { Cauliflower } \\
\text { Cabbage } \\
\text { Capsicum } \\
\text { Sunflower }\end{array}\right\}$ & $\begin{array}{l}\text { Large } \\
(\mathrm{I} \cdot \mathrm{O}-8 \cdot \mathrm{o} \mathrm{mm} \text { diam. })\end{array}$ & S. sclerotiorum \\
\hline
\end{tabular}

\section{METHODS}

Details of the $\mathrm{I} 2$ isolates of Sclerotinia used are given in Table I. These were cultured either directly on $\mathrm{I} 0 \mathrm{ml}$ of potato-dextrose agar (PDA) in plastic Petri dishes ( $9 \mathrm{~cm}$ diam) or on boiled cellophane discs placed over the medium. Each dish was inoculated with three isolates placed at equal distances around the edge. All possible combinations of the 12 isolates were studied, with eight replications for each combination on agar and four on cellophane. The cultures were incubated at $20^{\circ} \mathrm{C}$ under fluorescent light of about $\mathrm{I} 60 \mathrm{~lx}$ and were examined daily over a period of 3 weeks. The experiment was repeated.

\section{RESULTS}

The following three types of reaction were observed when hyphae of different isolates approached each other on agar cultures (Table 2).

(i) Mycelia intermingled freely and no antagonism between the hyphae was apparent (Fig. I $a, b, c, d$ ). Hyphal fusions (anastomoses) were observed between different mycelia, particularly between the hyphae of the small-sclerotium producing isolates.

(ii) A white zone, which later became pigmented, developed between mycelia and could be seen without magnification (Fig. I $c, e$ ). Microscopic examination showed that this white zone was due to excessive branching of the hyphal tips of one or both colonies, but that it was more frequent at the periphery of only one of the colonies (Fig. 2a) (cf. Fig. $2 b$ ). Eventually the hyphal tips at the margins of both of the interacting mycelia lysed to give an unevenly pigmented zone along the region of association. The hyphae of the different isolates were sometimes in physical contact, especially in regions where mycelial growth was less abundant, as when the fungi were grown on cellophane discs placed over agar; the hyphal tips of one colony then attached themselves to and grew around the hyphae of the others (Fig. $2 c, d$ ). This 'predatory' action eventually resulted in the lysis of the hyphae of the less aggressive isolate.

(iii) A brown zone developed rapidly between colonies (Fig. I $d, e$ ); the intensity of this varied from very light to dark brown, depending on the isolates used. Upon microscopic examination it was found that the tips of the peripheral hyphae of one of the colonies readily lysed as the margins of the two mycelia intermingled. Lysis could also occur before the 
Table 2. Types of mycelial interactions occurring between Sclerotinia isolates

Cultures were incubated on PDA at $20^{\circ} \mathrm{C}$ and $160 \mathrm{~lx}$ for 8 days.

$\left.\begin{array}{ll}\text { Isolates } & \text { SM-SI, SM-S2, SM-P, SM-T } \\ \text { SM-SI } \\ \text { SM-S2 } \\ \text { SM-P } \\ \text { SM-T }\end{array}\right\} \begin{aligned} & \text { Reaction type (i) } \\ & \text { (mycelia intermingle } \\ & \text { freely without any in- } \\ & \text { compatible reactions) }\end{aligned}$

$\left.\begin{array}{l}\text { ST-LI } \\ \text { ST-L2 }\end{array}\right\} \quad$ Reaction type (ii) Reaction type (i)

ST-BI

$\mathrm{ST}-\mathrm{B} 2$

SS-CLI

SS-CB

SS-CP

SS-SU
ST-LI, ST-L2

Reaction type (ii)

(formation of a white

Zone); ST-LI and ST-L2

'predatory' on SM isolates when hyphal contacts occur

Reaction type (iii)
ST-BI, ST-B2, SS-CLI, SS-CB, SS-CP, SS-SU

Reaction type (iii) (rapid formation of a brown zone - intensity of zone variable); SM isolates 'predatory' on SS, ST-BI and ST-B2 isolates when hyphal contacts occur

Reaction type (ii); ST-LI and ST-L2 isolates 'predatory' on ST-BI, ST-B2 and ss isolates when hyphal contacts occur

Reaction type (i)

different mycelia made physical contact with one another. A 'predatory' type of interaction similar to that described in (ii) was also frequently observed (Fig. $2 e$ ).

\section{DISCUSSION}

The type of reaction which occurs between mycelia of fungal isolates is probably an indication of their genetic relationship. Intermingling of hyphae without incompatibility and accompanied by anastomoses suggests a very close relationship. The development of a white zone due to excessive hyphal branching (reaction type ii) was probably induced by accumulation of substances in the substrate ahead of the opposing colonies. Eventual lysis of the hyphal tips occurred in both colonies and was probably the result of the adverse effects of the accumulating substances on the metabolism of the hyphae. This reaction indicated possible genetic incompatibility between the isolates. Reaction type (iii) also indicated metabolic and thus possible genetic differences between the mycelia involved. The 'predatory' growth of one hypha around another was obviously an antagonistic reaction, as it invariably resulted in the death of the hypha which was 'attacked'. Sclerotinia trifoliorum was always the 'predator' when grown with the other two species; when $S$. minor and S. sclerotiorum were grown together, the former was usually the 'predator' when physical contact was achieved.

The mycelial interactions observed during this investigation can be used to separate the I 2 selected isolates into three groups. Group I comprises isolates SM-P, SM-T, SM-SI and SM-S2, all of which are small-sclerotium forms obtained from miscellaneous crops. Group 2 comprises isolates ST-LI and ST-L2, which produce large sclerotia and were originally obtained from forage legumes (lucerne). Group 3 comprises large-sclerotium forming isolates ST-BI and ST-B2 obtained from vegetable legumes (beans), and SS-CLI, SS-CB, SS-CP and ss-sU from miscellaneous crops. This grouping agrees, without exception, with that which we obtained from the use of ontogenetic and electrophoretic criteria (Willetts \& Wong, 197I ; Wong \& Willetts, 1973). Further support is therefore given for the separation of $S$. sclerotiorum into three species, $S$. minor (exemplified by the isolates included above in group 1), S. trifoliorum (group 2) and S. sclerotiorum (group 3). 
(a)
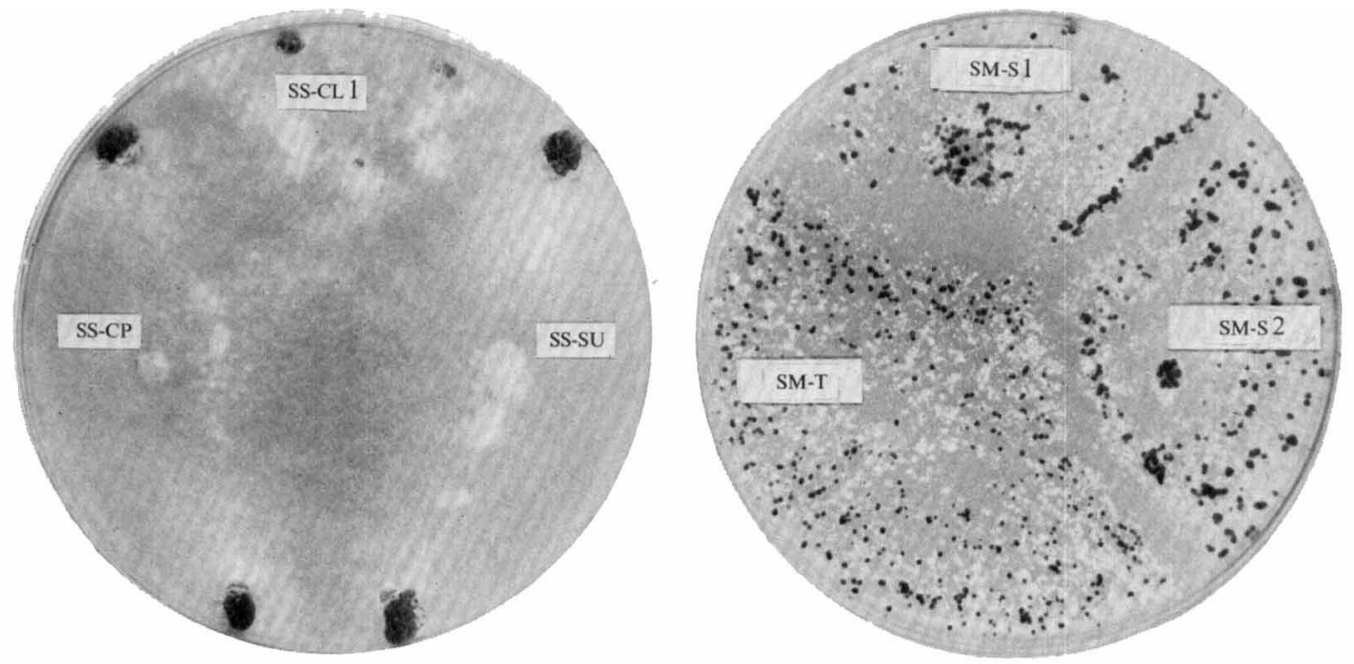

(b)

(c)

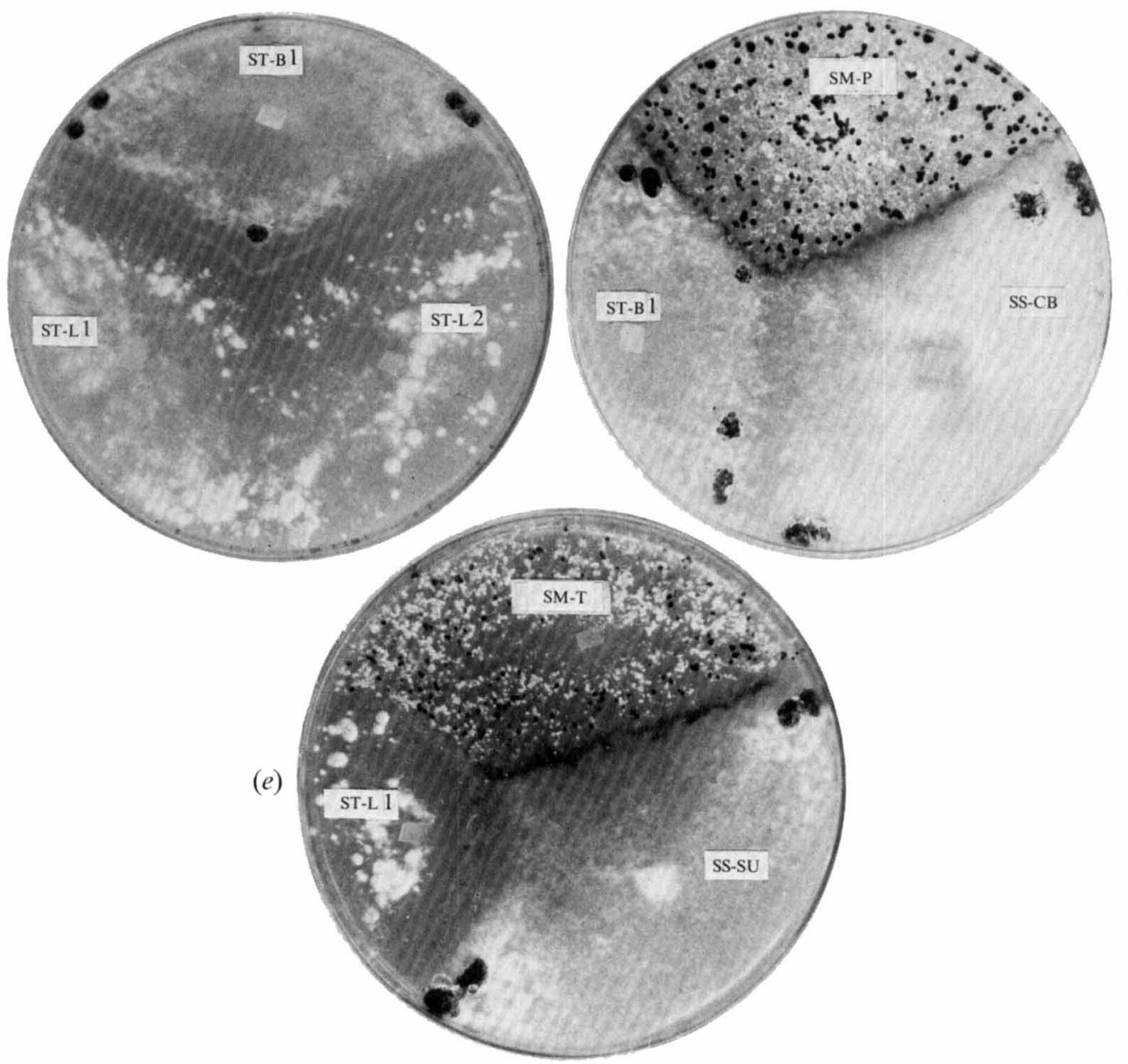

(d)

Fig. I. Reactions between Sclerotinia isolates at regions of association on agar-plate cultures. $(a),(b)$ Reaction between compatible isolates showing no antagonism: $(a)$ between ss isolates; $(b)$ between SM isolates. $(c)$ Reaction between ST-LI and ST-L2, showing no antagonism, and reaction of ST-LI and ST-L2 with ST-BI, resulting in the formation of a white reaction zone. (d) Reaction between ST-BI and SS-CB, showing no antagonism, and reaction of ST-BI and SS-CB with SM-P, resulting in the formation of a brown reaction zone. (e) Reaction of SS-SU and SM-T with ST-LI, resulting in the formation of a white reaction zone, and reaction between SS-SU and SM-T, resulting in the formation of a brown reaction zone. 

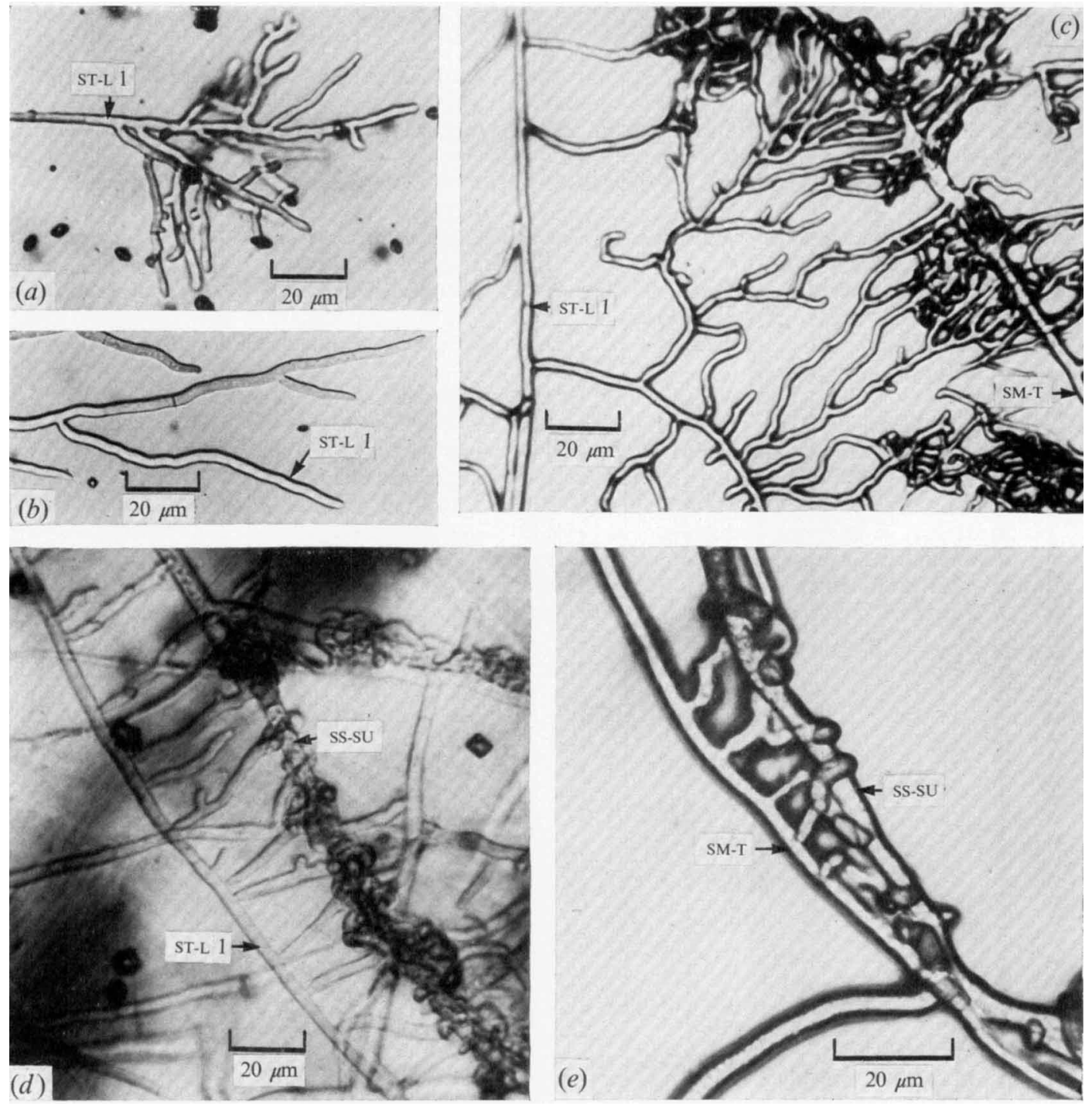

Fig. 2. Microscopic observations of excessive hyphal branchings and 'predatory' interactions at regions of association between Sclerotinia isolates. (a) Excessive hyphal branching reaction of ST-LI when approaching the mycelial front of SM-T in agar. (b) Normal hyphal tips and branchings of ST-L I at mycelial front. $(c),(d),(e)$ 'Predatory' action of hyphal tips of one isolate on the hyphae of another: $(c)$ 'predation' of SM-T by ST-LI ; $(d)$ 'predation' of SS-SU by ST-LI ; $(e)$ 'predation' of SS-SU by SM-T.

Co-inoculation techniques could have practical application in specific identifications when standard, known species are available. It is always necessary to make careful microscopic observations of the periphery of colonies as weak interactions could be overlooked or misinterpreted in a macroscopic examination. However, final identification, may require an electrophoretic analysis of protein extracts from the fungi.

We thank Drs R. J. W. Byrde, M. Marsden and A. E. Ashford for their critical reading of the manuscript, and the Reserve Bank of Australia for financial support. 


\section{REFERENCES}

BJörlıNG, K. (195I). Über die Entwicklungsgeschichte, Variabilität und Pathogenität von Sclerotinia trifoliorum Erikss. Phytopathologische Zeitschrift 19, I 29-1 56.

JAGGER, I. C. (1920). Sclerotinia minor, n.sp., the cause of a decay of lettuce, celery and other crops. Journal of Agricultural Research 20, 331-333.

KeAY, M. A. (1939). A study of certain species of the genus Sclerotinia. Annals of Applied Biology 26, 227246.

Korf, R. P. \& Dumont, K. P. (1972). Whetzelinia, a new generic name for Sclerotinia sclerotiorum and $S$. tuberosa. Mycologia 64, 248-251.

LOVELESS, A. R. (195I). The confirmation of the variety fabae Keay of Sclerotinia trifoliorum Eriksson. Annals of Applied Biology 39, 252-275.

Purdy, L. H. (1955). A broader concept of the species Sclerotinia sclerotiorum based on variability. Phytopathology 45, 421-427.

ReinHardt, M. O. (I 892). Das Wachsthum der Pilzhyphen. Ein Beitrag zur Kenntniss des Flächenwachsthums vegetabilischer Zellmembranen. Jahrbuch für wissenschaftliche Botanik 23, 509-5I3. [Reprinted in Buller, A. H. R. (1958). Researches on Fungi, vol. 5. New York: Hafner Publishing.]

WilletTs, H. J. \& Wong, A.-L. (197I). Ontogenetic diversity of sclerotia of Sclerotinia sclerotiorum and related species. Transactions of the British Mycological Society 57, 515-524.

Williams, G. H. \& WeSTERN, J. H. (1965). The biology of Sclerotinia trifoliorum Erikss. and other species of sclerotium-forming fungi. I. Apothecia formation from sclerotia. Annals of Applied Biology 56, 253-260.

Wong, A.-L. \& WilleTts, H. J. (1973). Electrophoretic studies of soluble proteins and enzymes of Sclerotinia species. Transactions of the British Mycological Society 6r, 167-178. 